\title{
A device for reducing the siltation of the front chamber of the pumping station in irrigation systems
}

\author{
Boborakhim Urishev ${ }^{1 *}$, Sobir Eshev, Fakhriddin Nosirov ${ }^{2}$, and Ulugbek Kuvatov ${ }^{1}$ \\ ${ }^{1}$ Karshi engineering-economics institute, 180100, Mustaqillik ave., Karshi, Uzbekistan \\ ${ }^{2}$ Termez branch of the Tashkent State Technical University named after Islam Karimov, 190100, \\ Islam Karimov st., Termez, Uzbekistan
}

\begin{abstract}
It has been determined that the reduction of siltation of the front chamber is necessary because the deposited sediments seriously violate the planned hydraulic structure of the flow when water is sucked up by pumps, as a result of which their efficiency decreases. The method of calculating the pipeline system of a new device designed to significantly reduce the siltation of the front chamber of irrigation pumping stations by artificially creating turbulence in the water flow in the bottom layer of the structure is presented. This calculation methodology is based on the use of the theory of flooded water jets, which makes it possible to determine the main characteristics of the jet. The latter allows calculating the design parameters of the pipeline system. The results of experimental studies are presented to compare the calculated data with the results of experiments, as well as to determine the effectiveness of the proposed device for a significant reduction in siltation of the front chamber of irrigation pumping stations.
\end{abstract}

Keywords: determined, water intake, flow, turbulent, restless character.

\section{Introduction}

Nowadays, the task of increasing the energy efficiency of pumping stations, ensuring their reliability in terms of guaranteed water supply to consumers with minimal losses of resources are the most important scientific and technical tasks [1,2]. Many pumping stations that operate in the irrigation system in agriculture of the Republic of Uzbekistan are forced to pump suspended (turbid) water formed as a result of erosion processes in the channels of supply canals and sources. As a result, in the front chamber of pumping stations, where the water velocities are minimal, sediments are deposited in thick layers, partly reaching a thickness of several meters, almost blocking the path to the extreme suction pipelines. This circumstance in the front chamber negatively affects the operating parameters of the pumps, their productivity decreases, the efficiency decreases, and the energy consumption increases. Observations have established that excessive sediment deposition in front of the suction pipelines leads to a decrease in the efficiency of the pump to $3.0 \%[3-20]$.

\footnotetext{
* Corresponding author: bob urishev@, mail.ru
} 
The movement of suspended sediment particles in a flow is complex, and it occurs under the influence of pulsation velocities that are variable in magnitude and direction, as well as under the action of gravity. If the force of gravity prevails due to certain circumstances, the particle can sink to the bottom, but when a sufficient vortex potential appears in the bottom layer, it is captured by it and rises again into the flow [6-12].

Depending on the transporting capacity of the water supply facilities, the water velocity may decrease to a value less than the non-lagging value (this is mainly in the front chamber and water intake chambers), as a result of which suspended particles are deposited at the bottom of the structures. This leads to a change in the design dimensions of the forechamber, clogging of the water intake chambers, which negatively affects the operational parameters of the pumping units [5, 21-34].

Under these circumstances, it becomes necessary to develop a device that allows artificially changing the structure of the flow in its bottom layer by multi-jet supply of pressurized water to prevent sediment deposition. Based on this, the main goal of the study was formulated, which consists in determining the pressure-flow characteristics of the device that provides a sufficient vortex state of the flow at the bottom of the fore-chamber of the pumping station, which does not allow sedimentation of sediment particles.

\section{Methods}

In order to overcome this issue, we have proposed a new design of the intake structure, which is equipped with an additional device that makes it possible significantly complicate sedimentation at the bottom of the fore-chamber $[13,19]$.

An additional device consists of a pressure supply pipe, which is connected on one side with the discharge pipeline of the pumping unit, and its other side has a special device in the form of annular perforated pipes laid along the slopes or the bottom of the fore-chamber in those places where sediments settle. Due to the hydrodynamic pressure created in the perforated pipes, water flows out of the pipe openings in a stream, and this circumstance creates a vortex flow in the medium that does not allow sedimentation. Thus, the flow in front of the units always has a turbulent, restless character, and the sediments will be in a suspended state and, together with water, are pumped through the suction pipe to the headwater (Fig. 1).

The main task of the device is to create a turbulent environment in the bottom layer of the flow, which does not allow sedimentation. This work must be performed by jets of water flowing out of the holes of the perforated pipes under the action of the hydrodynamic pressure created in them.

The issues of investigation of flooded water jets, which are used for different purposes, for example, for stirring up deposited sediments, are considered in [14, 15]. The main characteristics of the jet are determined, and the conditions for its interaction with the environment are clarified.

When water flows out of the pipe with an increase in the longitudinal dimensions of the jet, its transverse dimensions also increase. A turbulent jet boundary layer is formed at the jet boundary and near it. In this case, the arising intense velocity pulsations and mixing lead to the fact that between the jet and the surrounding flow there is an exchange of momentum, due to which the jet carries with it a part of the surrounding liquid, and in the zone of the jet outflow it is possible to observe a vortex (whirlpool) motion, which as an increase in the distance from the hole is gradually attenuated by separate whirlpools caused by transverse diffusion of mechanical energy [5, 16, 17].

Fig. 2. shows a diagram of the outflow of water from a pipe laid at the bottom of the intake structure.

The main parameters characterizing the jets are as follows. 
a) the value of the maximum jet velocity $u_{\text {max }}$ at a distance $x$.

b) the rate of flow of water from the hole, $u_{0}$.

c) c) diameter of the outflow hole, $d_{0}$.

d) the radius of the jet $R$ at a distance $x$.

The quantity $u_{\max }$ and $R$ G. N. Abramovich proposes to define by the formulas below [18].

$$
\begin{aligned}
& u_{\text {max }}=\frac{0.96 \cdot u_{0}}{\frac{2 a \cdot x}{d_{0}}+0.29} \\
& R=\left(\frac{6.8 a \cdot x}{d_{0}}+1\right) \frac{d_{0}}{2}
\end{aligned}
$$

Where $a$ - flow structure coefficient, this value for a round jet is taken to be $a \approx 0.08$. [18].

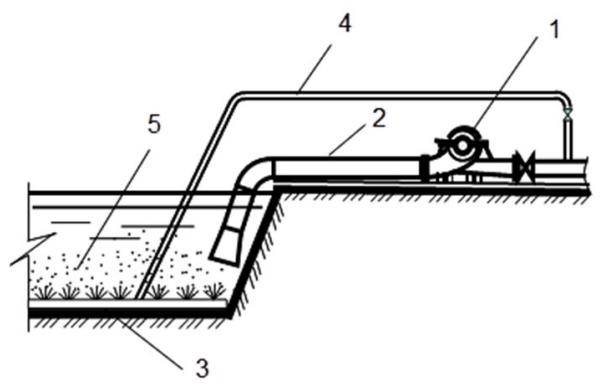

Fig. 1. Device for reducing siltation of the pumping station front chamber: 1 - pump; 2 suction pipe; 3 - avancamera; 4 - pipe for supplying water to the device; 5 - suspended sediment.

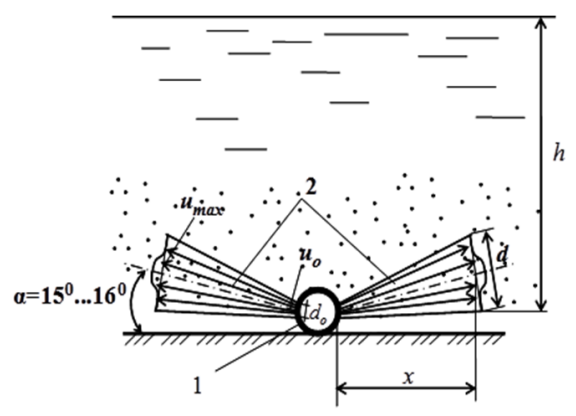

Fig. 2. Scheme of water flow from a perforated pipe: 1 -perforated pipe; 2 -water jets.

In order to ensure the free outflow and movement of the water jet, the side holes are made at an angle of tangent equal to half the jet expansion angle, i.e. $\operatorname{tg} \alpha=3.4 \cdot \mathrm{a}=0.272$, hence $\alpha=15^{0} \ldots 16^{0}$.

The flow rate of water from the hole is calculated by the formula

$$
u_{0}=\mu_{0} \sqrt{2 g H}
$$

Where $\mu_{0}$ - consumption coefficient; $H$ - the value of the excess pressure in the place of water outflow from the pipe over the pressure $\mathrm{h}$ in the water intake (in the front chamber).

$$
H=H_{1}-h
$$

where $\mathrm{H}_{1}$ is the pressure at the point where water flows out of the pipe.

In order for there to be no sedimentation in the front chamber, the value of the velocity $\mathrm{u}_{\max }$ at a distance $x$ from the hole must be greater than the non-wading velocity $\mathrm{u}_{\mathrm{z}}$.

The distance $\mathrm{x}$ from the hole at which $\mathrm{u}_{\max } \geq \mathrm{u}_{\mathrm{z}}$ is achieved can be determined in accordance with (1) at $a \approx 0.08$. next addiction

$$
x=\frac{1.81 d_{0}\left(3.31 u_{0}-u_{\max }\right)}{u_{\max }}
$$


Based on the above formula for $a \approx 0,08$ the diameter of an axisymmetric jet at a distance $x$ is determined by the following formula

$$
d=d_{0}+0.544 \cdot x
$$

\section{Results and discussion}

For maximum efficiency of creating a turbulent medium by water jets from perforated pipes, it is proposed to take the distance between them equal to $\mathrm{x}$, and the distance between the holes is equal (Fig. 3).

With such an arrangement of perforated pipes and their holes, it becomes possible to maximize the use of the hydrodynamic force of the jet for the formation of a turbulent state of the flow.

The diameters of the supply and perforated pipes are selected based on the passage of the maximum water flow through their cross-section while observing the economical value of the water velocity.

Determination of the maximum water flow through the supply pipeline can be carried out according to the following dependence

$$
Q=n_{1} \cdot n_{2} \cdot u_{0} \cdot \omega_{0}
$$

where $\mathrm{n}_{1}$ is the number of perforated pipes: $\mathrm{n}_{2}$ - number of holes in a perforated pipe: $\omega_{0}$ is the cross-sectional area of the holes.

The number of branches of perforated pipes is determined based on the possibility of their placement within the area where flow turbulization is recommended, taking into account the exclusion of the ingress of hydroabrasive particles into the water-conducting path of the pumping unit (Figure 4.). The results of our research on this issue showed that this section is located at a distance $l_{1}=(5 \ldots 6) D_{l}$ from the inlet part of the suction pipes, where there are usually no deposits of hydroabrasive particles.
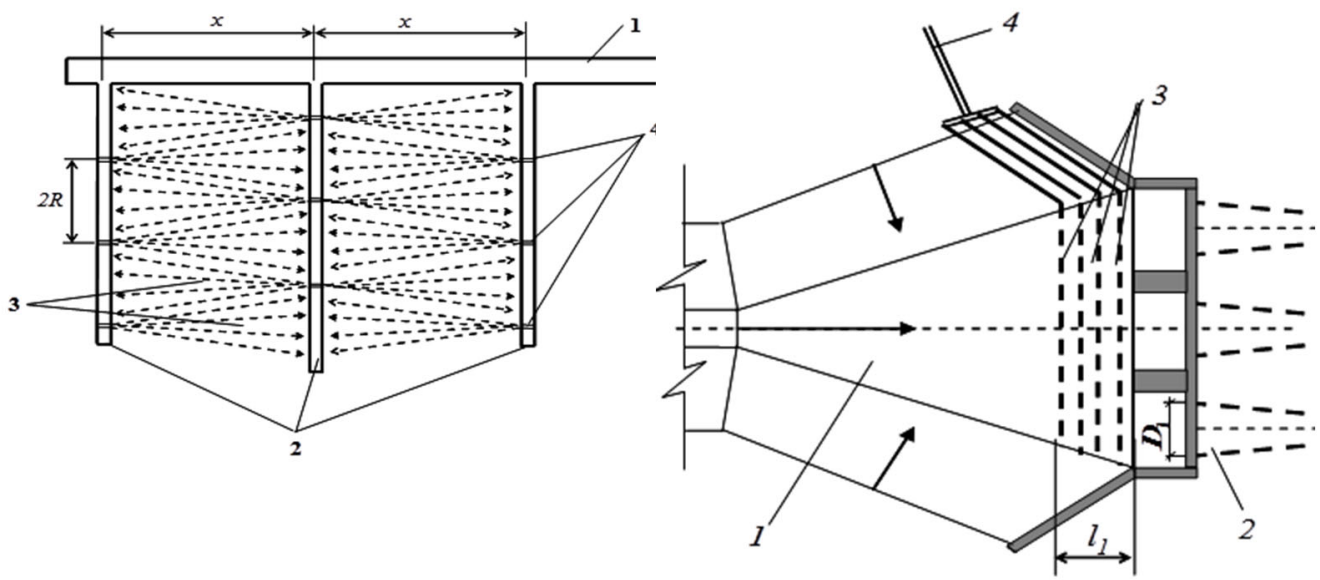

Fig. 3. Perforated pipe layout diagram: 1 supply pipe; 2 - perforated pipes; 3 approximate areas of action of the water jet; 4 holes in pipes.

Fig. 4. The layout of the perforated pipes in the front chamber of the pumping station «Sputnik»: 1 - avancamera; 2 - suction pipe; 3 - perforated pipes; 4 - supply pipe.

In this regard, the number of branches of perforated pipes is calculated by the following formula 


$$
n_{1}=\frac{6 \cdot D_{i n l}}{x}=\frac{6 \cdot D_{i n l}}{1.81 \cdot d_{0} \cdot k}=\frac{3.32 \cdot D_{1}}{d_{0} \cdot k}
$$

where, $x=1.81 \cdot d_{0} \cdot k, \quad k=\left(3.31 u_{0}-u_{\max }\right) / u_{\max }, D_{l}$ - diameter of the inlet part of the suction pipe.

The holes in the perforated pipe will be placed in the sections located at a distance $d=$ $2 \mathrm{R}$ from each other. In this regard, and taking into account that there are two holes in each section, their number in a perforated pipe can be determined by the following relationship

$$
n_{2}=\frac{2 \cdot l_{t r}}{d_{0}+0.544 \cdot x}=\frac{2 \cdot l_{t r}}{d_{0}+0.985 \cdot d_{0} \cdot k}
$$

where, $l_{\text {tr }}$ is the length of the perforated pipe. Thus, the water flow rate in the supply pipeline is calculated using the formula below

$$
Q=n_{1} \cdot n_{2} \cdot u_{0} \cdot \omega_{0}=\frac{2 \cdot 3.32 \cdot D_{1} \cdot l_{t r} \cdot u_{0} \cdot \omega_{0}}{d_{0}^{2} \cdot k(1+0.985 \cdot k)}=\frac{14.33 \cdot D_{1} \cdot l_{t r} \cdot \sqrt{H}}{k(1+0.985 \cdot k)}
$$

where, $u_{0}=\phi \sqrt{2 g H}=2.75 \sqrt{H}$ at $\varphi=0.62$, the cross-sectional area of the hole is as follows $\omega_{0}=0.785 \cdot d_{0}^{2}, H$ - water pressure in the supply pipe.

The maximum water flow rate taken by each perforated pipe from the supply pipeline based on (10) can be determined using the following formula

$$
Q_{1}=n_{2} \cdot u_{0} \cdot \omega_{0}=\frac{4.32 \cdot l_{t r} \cdot d_{0} \cdot \sqrt{H}}{1+0.985 \cdot k}
$$

To assess the proposed methodology, we studied the results of observations of the silting process in those areas of the front chamber where the perforated pipes were installed. The results of measuring the flow rate of water in the supply and perforated pipes showed that the deviation of the calculated data from the measured values does not exceed $7.0 \ldots 8.0 \%$.

Along with this, studies were carried out in the front chamber of the Sputnik pumping station in the Karshi main canal to determine the effectiveness of the proposed device (Fig. $5)$.

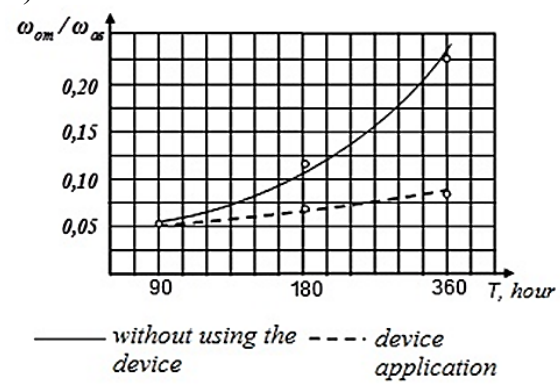

Fig. 5. Graphs of the dependence of $\omega_{\text {from }} / \omega_{\mathrm{av}}$ on the operating time of the pumping station $\mathrm{T}$.

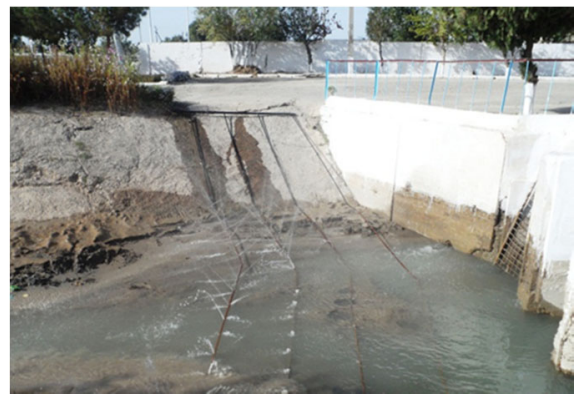

Fig. 6. Testing of a device to reduce siltation of the front chamber of the pumping station «Sputnik».

As the research results show, without the use of the device, the relative area of silting $\omega_{\text {from }} /$ $\omega_{a v}$ ( $\omega$ from is the area occupied by sediments, $\omega_{\mathrm{av}}$ is the total area of the front chamber) during the observation period increased to $25 \%$, and when using the device, the process of silting slowed down very noticeably, its intensity $8 \%$.

Checking the effectiveness of the device for reducing siltation of the front chamber of the pumping station «Sputnik», created on the basis of the results of calculations according 
to the above method, showed that, while ensuring the calculated pressure and flow parameters of the supplied water, the zone of action of the jets covers $86 \%$ of the area where the perforated pipes were laid (Figure 6). During the period of operation of the device, the usual structure of the flow in the bottom layer changed, the turbidity of the pumped water increased to $8.2 \mathrm{~kg} / \mathrm{m}^{3}$ (during the operation of three units before the application of the above device, the turbidity value was within $2.8 \ldots 3.2 \mathrm{~kg} / \mathrm{m}^{3}$ ).

\section{Conclusions}

1. A new device has been proposed to prevent siltation of the front chamber of the pumping station, which, according to the results of experimental studies, has shown high efficiency, determined by the fact that no sedimentation was observed on $83 \%$ of the area in the zone of the device.

2. A method is proposed for determining the parameters of the flow rate of water supplied to the pipeline system of the device to prevent siltation of the pumping station advance chamber. Comparison of the results of calculations by this method with experimental data showed that the discrepancy between them does not exceed $7.0 \ldots 8.0 \%$.

\section{References}

1. B. Urishev, Selection of Parameters of Pumped Storage Power Plants at Large Pumping Stations for Water Use, Applied Solar Energy, 54(6), 477-480 (2019), DOI: 10.3103/S0003701X18060166

2. B. U.Urishev, U. J. Kuvatov, Determination of Optimum Operational Parameters of Pump Stations, International Journal of Advanced Research in Science, Engineering and Technology, 6(4), 8890-8892 (2019)

3. B. Urishev, F. Artikbekova, D. Kuvvatov, F. Nosirov, U. Kuvatov, Trajectory of sediment deposition at the bottom of water intake structures of pumping stations, IOP Conf. Series: Materials Science and Engineering, 1030 (2021) DOI: 10.1088/1757899X/1030/1/012137

4. M. Muhammadiev, B. Urishev, S. Juraev, A. Boliev, Detritus removal from a pumpingplant intake chamber with hydrajet pumps, IOP Conf. Series: Materials Science and Engineering, 883 (2020) DOI: 10.1088/1757-899X/883/1/012123

5. M. M. Mukhammadiev, B. U. Urishev, F. Nosirov, Sedimentation of sediment particles in the pump station advance chamber, Hydrotechnical construction, 10, 33-36 (2012)

6. R. M. Dorrell, A. J. Hogg, E. J. Sumner, P. J. Talling, The structure of the deposit produced by sedimentation of polydisperse suspensions, Journal of geophysical research, 116 (2011) DOI: 10.1029/2010JF001718

7. G. K. Batchelor, Sedimentation in a dilute polydisperse system ofinteracting spheres, General theory, Fluid Mech., 119, 379-408 (1982) DOI: $10.1017 / \mathrm{S} 0022112082001402$

8. R. Н. Davisб Н. Gecolб Hindered settling function with noempirical parameters for polydisperse suspensions, AIChE, 40(3), 570-575, (1994) DOI: 10.1002/aic.690400317

9. A. S. Kondratev. Generalized method for determining the speed of restricted traffic monomodal gas bubbles, drops of liquid and solid particles of any shape under the influence of gravity, MAMI, 1(19), 4, 71-76 (2014)

10. R. M. Dorrell, A. J. Hogg, E. J. Sumner, P. J. Talling, The structure of the deposit produced by sedimentation of polydisperse suspensions, Journal of geophysical research, 116, (2011) DOI: 10.1029/2010JF001718

11. A. Sarimeseli, G. Kelbaliyev, Deposition of dispersed particles in isotropic turbulent flow, Dispersion Science and Technology, 29, 307-316 (2008) 
12. A. Altunbas, G. Kelbaliyev, K. Ceylan, Eddy diffusivity of particles in turbulent in rough channels Aerosol Science, 33, 1075-1084 (2002)

13. M. M. Mukhammadiev, B. U. Urishev, F. Zh. Nosirov, Water intake structure. Patent RUzb FAR 00238 (2006)

14. L. S. Zhivotovsky, L. A. Samoilovskaya, Technical mechanics slurry and dredge pumps (Moscow, 1986)

15. N. N. Kozhevnikov, Application and improvement of ejection devices of dredgers, Hydrotechnical construction, 1, 28-32 (1995)

16. A. Sarimeseli, G. Kelbaliyev, Sedimentation of solid particles in turbulent flow in horizontal channels, Powder Technology, 140, $79-87$ (2004)

17. A. Y. Varaskin, Y. V. Polezhaev, A. F. Polyakov, Effect of particle concentration on fluctuation velocity the disperse phase for turbulent pipe flow, Heat and Fluid Flow, 21, 562-574 (2000)

18. S. Eshev, Sh. Latipov, A. Qurbonov, J. Sagdiyev, M. Berdiev, N. Mamatov, Noneroding speed of water flow of channels running in cohesive soils, IOP Conf. Series: Materials Science and Engineering, 1030 (2021) DOI: 10.1088/1757899X/1030/1/012131

19. S. Eshev, A. Rakhimov, I. Gayimnazarov, A. Isakov, B. Shodiev, F. Bobomurodov, Dynamically stable sections of large soil canals taking into account wind Waves, IOP Conf. Series: Materials Science and Engineering, 1030012134 (2021) DOI: 10.1088/1757-899X/1030/1/012134

20. D. Bazarov, N. Vatin, B. Obidov, O. Vokhidov, Hydrodynamic effects of the flow on the slab of the stand in the presence of cavitation, IOP Conf. Ser. Mater. Sci. Eng., 1030, $012110(2021)$

21. D. Bazarov, I. Markova, B. Norkulov, O. Vokhidov, Hydraulic aspects of the layout of head structures during water intake from lowland rivers, IOP Conf. Ser. Mater. Sci. Eng. 1015, 012041 (2021)

22. B. Obidov, O. Vokhidov, D. Tadjieva, U. Kurbanova, A. Isakov, Hydrodynamic effects on the flow elements of the downstream devices in the presence of cavitation, IOP Conf. Ser. Mater. Sci. Eng., 1030, 012114 (2021)

23. D. Bazarov, B. Norkulov, O. Vokhidov, F. Uljaev, Z. Ishankulov, Two-dimensional flow movement in the area of protective regulatory structures, IOP Conf. Ser. Mater. Sci. Eng., 890, 012162 (2020)

24. D. Bazarov, I. Markova, S. Sultanov, F. Kattakulov, Dynamics of the hydraulic and alluvial regim e of the lower reaches of the Amudarya after the commissioning of the Takhiatash and Tuyamuyun hydrosystems, IOP Conf. Ser. Mater. Sci. Eng., 1030, $012110(2021)$

25. D. Bazarov, O. Vokhidov, Extinguishing Excess Flow Energy in Spillway Structures, in Proceedings of EECE 2020, LNCE 150, 535-545 (2021) DOI: 10.1007/978-3-03072404-7_52

26. A. Krutov, B. Norkulov, D. Mavlyanova, Simulation of spreading of non-conservative passive substances in water bodies, IOP Conf. Ser. Mater. Sci. Eng.m 883(1), 012028 (2020)

27. A.R. Khafizov, F. F. Kamaletdinov, A. B. Yakushkina, I. V. Nedoseko, Construction regulatory systems to protect the banks of the Ufa river in the area of Ufa water intake, Izvestiya KGASU, 1(51), 118-127 (2020)

28. R. Kh. Mukhametrakhimov, I. R. Aliullova, Improvement of the quality control system for expansion joints with rubber compensators during the repair of bridge constructions, Izvestiya KGASU, 3(53), 47-55 (2020) 
29. R. Kh. Mukhametrakhimov, A. A. Panchenko, Features of the quality control system for the construction of outdoor water supply and sewerage networks, Izvestiya KGASU, 4(42), 360-367 (2017)

30. A. Krutov, B. Norkulov, P. Nurmatov, M. Mirzaev, Applicability of zero-dimensional equations to forecast nonconservative components concentration in water bodies, IOP Conf. Ser. Mater. Sci. Eng., 883(1), 012028 (2020)

31. A. Krutov, B. Norkulov, F. Artikbekova, P. Nurmatov, Optimal location of an intake at a reservoir prone to salt diffusion, IOP Conf. Ser. Mater. Sci. Eng., 869(7), 072020 (2020)

32. B. Matyakubov, I. Begmatov, I. Raimova, G. Teplova, Factors for the efficient use of water distribution facilities, IOP Conf. Ser. Mater. Sci. Eng., 883, 012025 (2020)

33. B. Uralov, N. Rakhmatov, S. Khidirov, F. Uljaev, I. Raimova, Hydraulic modes of damless water intake, IOP Conf. Ser. Mater. Sci. Eng., 1030(1), 012123 (2021)

34. D. Bazarov, I. Markova, I. Raimova, Sh. Sultanov, Water flow motion in the vehicle of main channels, IOP Conf. Ser. Mater. Sci. Eng., 883, 012025 (2020) 